\title{
PAOLO DRUDE
}

Il 5 luglio scorso una perdita grave e del tutto inaspettata colpiva la nostra scienza; in quel giorno Paolo Drude, che, quale titolare della cattedra di Fisica sperimentale nell' Università di Berlino e quale direttore degli Annalen der Physik, aveva conquistato una delle principali posizioni fra i fisici tedeschi, in un improvviso ottenebramento della mente, poneva da se stesso una fine prematura alla sua vita straordinariamente attiva e ricca di successo.

Drude nacque il 12 luglio 1863 in Braunschweig. Dal 1882 egli erasi dedicato allo studio delle Matematiche e della Fisica nelle Università di Berlino, Friburgo e Gottinga. Sotto la guida dei più illustri maestri quali Weierstrass, Kronecker, Kummer, Fuchs, Schwarz, Klein, e Helmholtz, Kirchhoff, Warburg, Riecke, Voigt, egli acquistó una coltura singolal’mente vasta e profonda. Però fra quelli fu il Voigt, il continuatore della celebre scuola di F. Neumann a Gottinga, colui che esercito un'azione decisiva sullo sviluppo scientifico del Drude; ed il Drude stesso volle con animo grato proclamarsi scolaro del Voigt anche nel discorso pronunziato in occasione della sua ammissione all'Accademia di Berlino.

La dissertazione dottorale del Drude (Sulle leggi della riflessione e della rifrazione della Iuce alla superficie dei cristalli assorbenti, 1887), lavoro teoretico eccellente, con cui egli inaugurò la sua carriera scientifica piena di promesse, si ricollega appunto al problema trattato in quel tempo dal Voigt della propagazione della luce nei mezzi assorbenti. A questo lavoro segui presto una ricerca sperimentale sulla riflessione nell'An- 
timonite, colla quale egli confermava i risultati della teoria. 11 latto osservato in quest'occasione che il comportamento ottico di una superficie di sfaldatura recente si altera per esposizione all'aria, condusse il Drude a profonde ricerche teoriche e sperimentali sopra l' influenza di strati superficiali, estranei, sulla riflessione della luce, ed egli potè cosi mostrare che la polarizzazione ellittica nella riflessione su corpi traspa. renti è dovuta a tali strati superficiali; con che veniva ad essere tolta un'apparente contraddizione tra l'esperienza e la teoria di F, Neumann. Grazie ai risultati ottenuti in questi lavori, il Drude potè passare a determinare, mediante misure di riflessione, le costanti ottiche dei metalli in modo molto più preciso e per una serie molto maggiore di corpi, che non fosse stato fatto sino allora. I risultati di questa ricerca sperimentale assai penosa sono raccolti nella sua dissertazione di libera docenza (1890).

Negli anni successivi il Drude pubblicava un gran numero di lavori di ottica, fra i quali ricordiamo solo una felice trattazione teorica dell'influenza, scoperta dal Kerr, della magnetizzazione sopra la riflessione sulla superficie del ferro, del cobalto, e del nickel, ed un' elegante esperienza, eseguita in collaborazione col Nernst, con cui fu dimostrato che l'eccitazione della fluorescenza di un'onda polarizzala è dovuta a vibrazioni normali al piano di polarizzazione.

Se nei suoi primi lavori il Drude era partito dai concetti della teoria meccanica della luce generalizzata dal Voigt, egli si approfondi ben presto anche nella teoria elettromagnetica, che in Germania cominció a trovare favore solo dopo le espevienze di Hertz, e nel 1902 egli potè dare un'eccellente e chitra esposizione del valore relativo delle diverse teorie per la descrizione matematica dei fenomeni luminosi. E da quel tempo noi vediamo il Drude diventare fautore e propugnatore sempre più ardente della teoria elettromagnetica. A questa sua attitudine ha molto contribuito il fatto che le lezioni piene di interesse tenute a Gottinga quale libero docente, sulle oscillazionj elettriche e sulla teoria elettromagnetica, lo condussero ad una conoscenza profonda di questo campo. Il suo libro « Fisica dell' etere » uscito nel 1904, rappresenta il frutto di questi 
suoi studi; in tale opera di maggior mole egli espose la teoria dell'elettricità e dell'ottica dal punto di vista del Maxwell, con chiarezza meravigliosa ed evitando calcoli estesi, e contribui molto allà diffusione della teoria del Maxwell in Germania.

Chiamato nell'autunno 1884 a straordinario di Fisica teorica a Lipsia, il giovane scienziato ottenne la posizione agognata ed il modo di sviluppare una larga attività di insegnante, a cui si dedicò con entusiasmo. Oltre a ciò, egli trovó tempo e mezzo ad un intenso lavoro sperimentale originale, principalmente sulle oscillazioni elettriche. Ed il problema della dipendenza della costante dielettrica dalla durata di oscillazione, che sta in stretto legame colla teoria elettro-magnetica della dispersione, fu per la prima volta trattato in modo sistematico. I metodi da lui immaginati in quest' occasione, gli apparecchi costruiti, danno una norma della sua straordinaria attitudine alla ricerca sperimentale esatta e sempre guidata da concetti teorici, e dimostrano la sua abilitá tecnica; il suo apparechio per la determinazione della costante dielettrica, nella sua ultima forma maneggevole ed atta a piccole quantità di sostanza, si è introdotta in tutti i laboratori di Fisica. Ma anche i risultati di quella grossa ricerca sperimentale sono importanti; egli mostrò che una serie di liquidi, nel campo di onde Hertziane brevi, presenta la dispersione anomala, ciò̀ una costante dielettrica, che diminuisce colla durata di oscillazione, e nello stesso tempo forte assorbimento - e che tale comportamento è legato alla presenza di certi gruppi atomici; ciò che permette di trarre delle conclusioni sulla costituzione chimica di tali liquidi.

Allorquando negli ultimi anni del secolo passato la scoperta degli elettroni liberi mostrò la possibilità di una spiega. zione soddisfacente della conduttività metallica, il Drude si volse nuovamente al problema del legame fra le proprietà elettriche e le proprietà ottiche dei metalli. E gli venne fatto non solo di rimuovere le contraddizioni, che sino allora parevano sussistere in questo argomento, ammettendo l'esistenza sia di elettroni vibranti intorno ad una posizione di equilibrio, sia di elettroni liberi di muoversí; ma egli potè anche, applicando con audacia i concetti della teoria cinetica dei gas al moto 
degli ioni liberi, istituire una teoria, che gli forni importanti relazioni del comportamento ottico col termico, come pure cogli effetti termoelettrici, termomagnetici e galvanomagnetici.

Un primo brillante successo di questa teoria pubblicata nel 1900 fu il calcolo del legame numerico fra la conduttivita termica e l'elettrica, ed i loro coefficienti di temperatura.

La sua teoria elettronica dei metalli, se pure dovrà subire qualche modificazione e qualche sviluppo ulteriore, rimarrà sempre fruttifera e di grande valore, non fosse che per i problemi, che essa pone all'indagine sperimentale. Oltre a questi grossi lavori teorici, il Drude portava nello stesso tempo a termine il suo trattato di Ottica, venuto a riempire nel modo più felice una lacuna nella letteratura fisica tedesca. L'esposizione chiara, la scelta opportuna degli argomenti trattati, l'attenzione ri. chiamata sui problemi moderni più importanti, l' avere tralasciato i calcoli più pesanti spiegano abbastanza il successo eccezionale ottenuto dal libro; tosto ne uscì una traduzione inglese e poco prima della sua morte l'autore poteva consegnare alla stampa il manoscritto della seconda edizione, in cui aveva tenuto conto dei più recenti progressi.

Alla felice produzione del Drude non poteva non seguire la considerazione pubblica. A lui fu affidata dopo la morte di G. Wiedemann la direzione degli Annalen der Physik, ufficio che egli occupó dal 1900 sino alla sua morte con generale soddisfazione, grazie al suo giudizio imparziale ed alle sue doti personali di cortesia. Poco dopo, nella primavera del 1900, egli segui l'invito di salire la cattedra di Fisica sperimentale a Giessen; ivi assunse con il più grande zelo e con pieno successo la direzione di un laboratorio sperimentale eccellentemente arredato. Numerosi scolari corsero a Giessen, chiamati dalla fama scientifica e dal valore didattico del Drude; ed un numero non piccolo di tesi sono uscite dall' Istituto di Giessen durante i 5 anni della sua direzione.

Ma dopo breve interruzione egli riprese $\mathrm{i}$ propri lavori. Da una parte si applicó a sviluppare ulteriormente la teoria elettronica dell'ottica specialmente circa la dispersione dei mezzi trasparenti; e gli venne fatto di calcolare dalla dispersione dell' idrogeno il rapporto fra la carica e la massa apparente 
degli elettroni negativi, trovando un valore vicino a quello ricavato per i raggi catodici; di più trovó un legame fra il numero degli elettroni otticamente efficaci e la valenza chimica, legame che promette di diventare molto importante. L'altro campo della sua attività in Giessen fu quella delle oscillazioni elettriche nei trasformatori Tesla. Facendo sempre camminare di pari passo la teoria e l'esperienza, egli ricercò in modo sistematico la disposizione più favorevole delle spirali; problema che presenta anche un grande interesse dal punto di vista pratico della telegrafia senza fili. Lo scopo, che egli proseguiva in questo argomento, era quello di mandare sull'antenna di trasmissione onde possibilmente pure e poco smorzate. Il Drude stesso aveva compilato una relazione comprensiva sui risultati ottenuti da lui e dai suoi scolari in questo campo, per presentarla all' ultima riunione dei naturalisti tedeschi a Stuttgart ed egli designava lo stesso argomento come uno dei temi, che si riservava di sviluppare, quando lesse il suo discorso di ammissione all' Accademia di Berlino, otto giorni prima della sua morte.

Il Drude stesso ricordava il periodo della sua attività a Giessen, come il più fortunato della sua vita. E quanto fosse soddisfatto della sua opera colà lo dimostra il fatto che egli rifiutò niente meno che 3 inviti di altre università (Tubinga, Lipsia, Breslavia) malgrado offerte certamente vantaggiose per lui. Ma quando per il passaggio di Warburg all' Istituto centrale imperiale di Charlottenburg, gli fu offerta la prima cattedra di Fisica in Germania, egli credette non potere sottrarsi alla straordinaria fiducia, che gli veniva dimostrata e passò nella Pasqua del 1905 a Berlino, non senza qualche esitazione. Con febbrile attivitá egli cercò subito di rendersi padrone della nuova situazione coi gravi obblighi inerenti alla scuola e all'amministrazione, per potere di nuovo dedicarsi ai propri lavori scientifici, i cui progetti egli espose nel discorso già citato all'Accademia di Berlino. Ma il cumulo di lavoro proveniente dal suo ufficio e dai doveri dalla nuova posizione, dalla direzione che egli conservò degli Annalen der Physik si mo. strò eccessivo anche per una tempra eccezionale di lavoratore, quale aveva il Drude e produsse in lui una perturbazione ner. 
vosa, i cui segni non furono quasi palesi a quelli che non gli stavano vicino, ma che lo condusse finalmente alla catastrofe del 5 luglio.

A tutti coloro che avevano conosciuto il Irude, a coloro che, come lo scrittore di queste righe, lo avevano veduto nell'ultima primavera pieno della antica freschezza ed energia, la notizia della sua tragica fine parve incredibile; proprio a lui pareva dover essere riserbato il colmo della felicità umana, per il suo temperamento allegro, la sua forza di volontà, la sua salute di ferro, per il successo della sua attività, la stima dei suoi colleghi, la sua serena vita di famiglia.

La scienza piange la perdita di una simile forza recisa nel pieno del vigore, e nello stesso tempo conserva una memoria grata dell'uomo, che nell'opera di meno di 20 anni le lascia una cosi ricca produzione.

Federico Pockels. 